\title{
Effect of mesenchymal stem cells on Sjögren-like mice and the microRNA expression profiles of splenic $\mathrm{CD4}^{+} \mathrm{T}$ cells
}

\author{
GUANG-FENG RUAN $^{1 *}$, LING ZHENG ${ }^{2 *}$, JIA-SHU HUANG ${ }^{1}$, WAN-XUE HUANG ${ }^{1}$, \\ BANG-DONG GONG ${ }^{1}$, XING-XING FANG ${ }^{1}$, XIAO-YU ZHANG ${ }^{1}$ and JIAN-PING TANG ${ }^{1}$
}

Departments of ${ }^{1}$ Rheumatology and ${ }^{2}$ Respiratory Medicine, Tongji Hospital of Tongji University, Shanghai 200065, P.R. China

Received October 31, 2015; Accepted December 9, 2016

DOI: $10.3892 / \mathrm{etm} .2017 .4313$

\begin{abstract}
Mesenchymal stem cells (MSCs) serve immunoregulatory functions and offer a promising novel treatment for certain autoimmune diseases. The present study investigated the therapeutic effect of mice bone marrow (BM)-MSCs on mice with relatively late stage of Sjögren-like disease and the impact of BM-MSCs on the microRNA (miRNA) expression profiles of splenic $\mathrm{CD}^{+} \mathrm{T}$ cells. Female NOD/Ltj mice were randomized into two groups: The disease group $(n=8)$ and the MSC-treated group $(n=8)$. Female ICR mice served as the healthy control group $(n=8)$. The MSC-treated group received an injection of MSCs when they were 26 weeks old. Water intake, blood glucose and salivary flow rate were measured and submandibular glands were resected and stained with hematoxylin and eosin to calculate the focus score. The concentrations of interleukin (IL)-2, IL-6, hepatocyte growth factor, interferon $\gamma$, IL-10, prostaglandin E2, transforming growth factor $\beta 1$ and tumor necrosis factor- $\alpha$ in serum were measured using ELISA. The expression of miRNAs in splenic $\mathrm{CD}^{+} \mathrm{T}$ cells were measured using deep sequencing. The results demonstrated that treatment with BM-MSCs prevented a decline in the salivary flow rate and lymphocyte infiltration in the salivary glands of NOD mice, indicating that MSC-treatment had a therapeutic effect on NOD mice with relatively late stage of Sjögren-like disease. ELISA and deep sequencing results showed that the three groups of mice had different serum concentrations of cytokines/growth factors and different miRNA expression profiles of splenic $\mathrm{CD}^{+}$ $\mathrm{T}$ cells. This implies that the alteration in serum levels of cytokines/growth factors and miRNA expression profiles of
\end{abstract}

Correspondence to: Professor Jian-Ping Tang, Department of Rheumatology, Tongji Hospital of Tongji University, 389 Xincun Road, Shanghai 200065, P.R. China

E-mail: tangjp6512@126.com

*Contributed equally

Key words: Sjögren's syndrome, mesenchymal stem cells, cytokines/growth factors, $\mathrm{CD} 4^{+} \mathrm{T}$ cells, microRNAs splenic $\mathrm{CD}^{+} \mathrm{T}$ cells may explain the therapeutic effect MSCs have on Sjögren's syndrome.

\section{Introduction}

Sjögren's syndrome (SS) is a systemic autoimmune disease that primarily affects the exocrine glands. Primary clinical symptoms include dry eyes (keratoconjuctivitis sicca) and dry mouth (xerostomia); but extraglandular manifestations of the disease, such as cutaneous vasculitis, Raynaud phenomenon, arthritis, liver, lung, kidney and rarely peripheral nerve involvement may also occur (1). The histological hallmark of $\mathrm{SS}$ is focal lymphocytic infiltration of the exocrine glands, which is detected by minor labial salivary gland biopsy (2). Currently, the treatment of SS is challenging (3). CD $4^{+} \mathrm{T}$ cell is a type of $\mathrm{T}$ cell that serves an important role in the immune system, aiding the activity of other immune cells by releasing $\mathrm{T}$ cell cytokines (4). A number of studies have demonstrated that abnormal and dysregulated $\mathrm{CD} 4^{+} \mathrm{T}$ cells may serve a role in the pathogenesis of SS (5-11).

The non-obese diabetic (NOD) mouse is a frequently used animal model to study SS. It is also used to investigate type 1 diabetes mellitus, which exhibits infiltrates of lymphocytes in the salivary glands (sialadenitis) causing a gradual loss of salivary function and also infiltrates of lymphocytes in the pancreas (insulitis) (12-14). This reduced saliva output is similar to what occurs in patients with SS (12).

Mesenchymal stem cells (MSCs) are multipotent stem cells that have the capacity to differentiate into chondrocytes, adipocytes, osteoblasts and neural cells (15). MSCs are present in various tissues, including the teeth, muscle, skin, fat, bone marrow and umbilical cord (16). Following stimulation with cytokines in an inflammatory environment, MSCs secrete a variety of immunoregulatory molecules, growth factors and chemokines that can modulate $\mathrm{T}$ and $\mathrm{B}$ lymphocytes, dendritic cells and natural killer cells (17-20). Furthermore, MSCs express low levels of major histocompatibility complex (MHC) class I, but do not express MHC class II surface molecules and consequently cannot serve as effective antigen-presenting cells to promote an immune response (21). Therefore, their low immunogenicity and immunoregulatory potential may enable them to be developed as a novel treatment for SS (22). A number of experiments involving NOD mice have suggested that MSCs have therapeutic potential in treating SS (22-24). It has been 
demonstrated that MSCs exert immunoregulatory function partially by inhibiting the proliferation of $\mathrm{CD}^{+} \mathrm{T}$ cells and regulating proportion of the subset of $\mathrm{CD}^{+} \mathrm{T}$ cells, including Treg, Th2, Th17 and Tfh $(22,25)$. However, NOD mice in the experiments cited received MSC therapy between the ages of 7 and 16 weeks, which represents a relatively early stage of SS. By contrast, the therapeutic effect of MSC-treatment in mice with relatively late stage SS has not yet been tested.

MicroRNAs (miRNAs) are small non-coding RNAs 20-27 nucleotides long that suppress translation by undergoing imperfect base pairing with their target mRNAs (26). The non-coding RNA molecules serve a fundamental role in the regulation of gene expression, and it is estimated that $30 \%$ of all coding genes are potential targets of miRNAs (26). A number of studies have been conducted on the miRNAs of peripheral blood mononuclear cells (PBMCs) from patients with SS, as well as in NOD mice. The results showed that the miRNA profiles of PBMCs in SS are different from healthy controls in humans and mice with SS, and certain differentially expressed miRNAs, such as miR-146a and miR-155, are thought to contribute to the pathogenesis of SS (27-30).

Therefore, results from previous studies indicate that MSCs may help to prevent SS and restore salivary gland function in NOD mice with early stage SS. However, it remains unknown whether NOD mice with relatively late stage SS could also benefit from MSC treatment. MSCs can exert immunoregulatory function partly by regulating the proliferation and differentiation of $\mathrm{CD}^{+} \mathrm{T}$ cells; however, the underlying mechanisms of MSC action are not yet fully understood. The miRNA profiles of PBMCs from patients with SS and healthy controls differ and some of the differentially expressed miRNAs may serve roles in the pathogenesis of SS (27-30). Therefore, it is worth investigating whether MSCs can alter CD4+ $\mathrm{T}$ cells miRNAs profiles as an underlying mechanism to exert their therapeutic effect on NOD mice. The aim of the present study was to investigate the therapeutic effect of bone marrow (BM)-MSCs on mice with relatively late stage SS and identify the different miRNAs profiles of splenic $\mathrm{CD}^{+}$ $\mathrm{T}$ cells among three groups of mice: Healthy control mice, MSC-treated SS mice and untreated SS mice.

\section{Materials and methods}

Animal model. Female NOD/Ltj mice served as an SS animal model, and female ICR mice served as controls $(\mathrm{n}=8)$ and MSC donors $(\mathrm{n}=16)$. Six-week-old female NOD/Ltj $(\mathrm{n}=20, \sim 20 \mathrm{~g})$ and ICR mice $(n=28, \sim 20 \mathrm{~g})$ were purchased from Shanghai SLAC Laboratory Animal Co., Ltd. (Shanghai, China) and maintained under specific pathogen-free conditions with a $12 / 12 \mathrm{~h} \mathrm{light} /$ dark cycle $\left(22 \pm 3^{\circ} \mathrm{C}, 50 \%\right.$ humidity $)$, with food and water available ad libitum in the Experimental Animal Center of Tongji University (Shanghai, China). Female NOD/Ltj mice were randomized into two groups: An untreated group $(n=8)$ and a group treated with MSCs $(n=8)$. All protocols on animal use were approved by the Institutional Ethics Committee of the Experimental Animal Center of Tongji University.

BM-MSC isolation and enrichment. ICR donor mice (8-weeks-old; $n=16$ ) were sacrificed by cervical dislocation. Muscles were removed to expose the femur and tibia. Mouse
BM-MSCs were collected by flushing the femurs and tibias with Dulbecco's modified Eagle's medium (DMEM)/F12 (Gibco; Thermo Fisher Scientific, Inc., Waltham, MA, USA), which was supplemented with $20 \%$ fetal bovine serum (Gibco; Thermo Fisher Scientific, Inc.), $100 \mathrm{U} / \mathrm{ml}$ penicillin and $100 \mu \mathrm{g} / \mathrm{ml}$ streptomycin (Gibco; Thermo Fisher Scientific, Inc.). After the cells had been cultured at $37^{\circ} \mathrm{C}$ overnight, the culture medium was refreshed to remove non-adherent cells. All MSCs used in the experiment were derived from passages $3-5$.

Measurements of water intake, blood glucose and salivary flow rates of NOD/Ltj mice. The three groups mice were housed between the ages of 6 and 30 weeks. The drinking bottles of the rearing cage were weighed every day, and the weight difference of the bottles was the total water intake of mice in the cage (each cage contained 4 mice; only mice from the same group were kept in the same cage, and individual water intake was calculated by dividing the weight difference of the bottles by 4). Glucose levels in tail-vein blood samples were measured once a week using a blood glucometer (Sannuo; Changsha Sinocare, Inc., Changsha, China). Briefly, following sterilization of the tail with $75 \%$ ethanol, the tail-vein was cut gently using a sterile surgical blade until blood flowed out. The blood was then dropped onto glucometer test paper (Sannuo; Changsha Sinocare, Inc.), using the glucometer to read the blood glucose level. If the mice had hyperglycemia, intraperitoneal injection of long-acting insulin was administered once a day to control blood glucose. Blood glucose levels were measured directly prior to insulin injection. Salivary flow rates were obtained by intraperitoneal injection of $4 \%$ chloral hydrate (Yangzhou Aoxin Chemical Co., Ltd., Yangzhou, China; $10 \mathrm{ml} / \mathrm{kg}$ body weight) to induce anesthesia in the mice. Whole saliva was collected following stimulation of secretion by subcutaneous administration of pilocarpine (Shanghai Chengshao Biological Technology, Co., Ltd., Shanghai, China; $0.5 \mathrm{mg} / \mathrm{kg}$ body weight). Saliva was obtained from the cotton sliver stuffed in the mice mouth over a 10 -min period and saliva volume was measured gravimetrically (the cotton sliver was weighed before and after being placed in the mouse's mouth, the increase in weight was calculated to determine the volume, based on the assumption that the specific gravity was $1 \mathrm{~g} / \mathrm{ml})$. Salivary flow rates were determined when the mice were aged between 26 and 30 weeks old.

MSC infusion. For MSC treatment, 26-week-old female NOD/Ltj mice $(n=8)$ were injected with BM-MSCs $\left(5 \times 10^{5}\right.$ cells/mouse) in $0.4 \mathrm{ml}$ PBS via tail vein, twice a week for two consecutive weeks.

Focus score. Mice from the three groups ( $\mathrm{n}=8$ per group) were sacrificed at the age of 30 weeks by cardiac puncture following the intraperitoneal injection of $4 \%$ chloral hydrate to induce anesthesia in the mice. Following sacrifice, the submandibular glands of the mice were removed immediately. Samples were fixed with $4 \%$ paraformaldehyde at $4^{\circ} \mathrm{C}$ for $24 \mathrm{~h}$, then embedded in paraffin. Sections $10-\mathrm{mm}$ thick were cut and stained with hematoxylin and eosin. Focus scores were calculated as the number of foci (aggregates) of $\geq 50$ inflammatory 
A

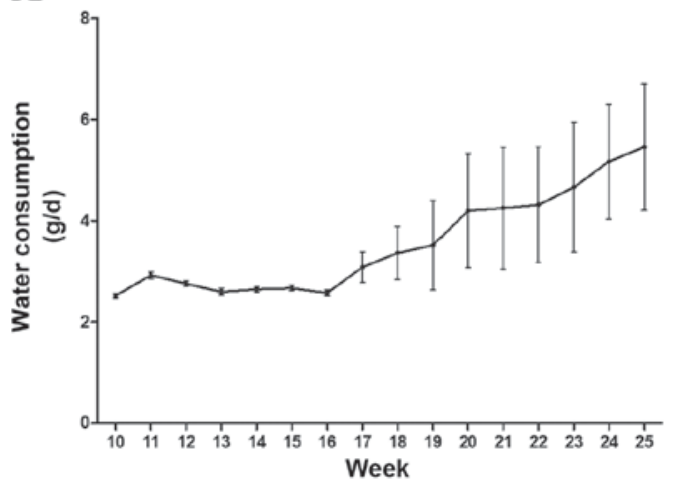

B

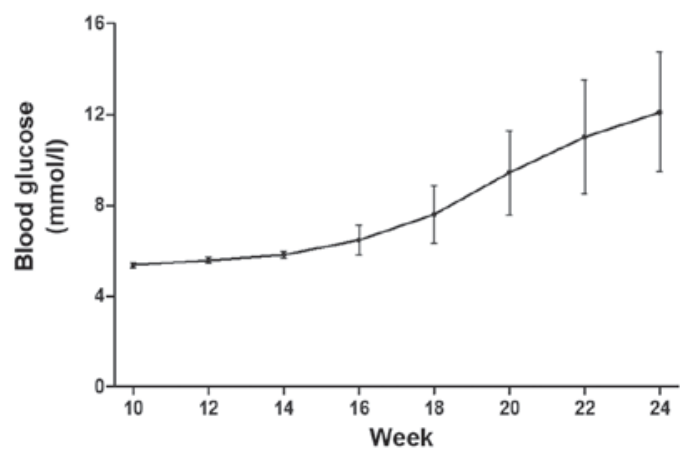

Figure 1. Daily water consumption and blood glucose levels of NOD mice. (A) Daily water consumption of NOD mice remained stable prior to week 16 and gradually increased after that $(n=16 ; \mathrm{P}<0.05)$. (B) Blood glucose levels of NOD mice also increased after 16 weeks ( $=16$; $\mathrm{P}<0.05)$. NOD mice, non-obese diabetic mice.

cells per $4 \mathrm{~mm}^{2}$ of salivary tissue. The score was calculated per field at x200 magnification using Image-Pro Plus version 6.0 software (Media Cybernetics, Inc., Rockville, MD, USA). A higher focus score indicated an increased level of inflammation in the salivary tissue. This method is an accepted way to determine the severity of sialadenitis (23).

ELISA. Mouse blood was drawn by cardiac puncture following anesthesia by $4 \%$ chloral hydrate, placed at $37^{\circ} \mathrm{C}$ for $2 \mathrm{~h}$ then at $4^{\circ} \mathrm{C}$ for $10 \mathrm{~h}$, and then centrifuged $(500 \times \mathrm{g}$ at room temperature for $10 \mathrm{~min}$ ) to obtain serum. The concentrations of the cytokines interleukin (IL)-2, IL-6, hepatocyte growth factor (HGF), interferon $\gamma($ IFN- $\gamma$ ), IL-10, prostaglandin E2 (PEG2), transforming growth factor $\beta 1$ (TGF- $\beta 1$ ) and tumor necrosis factor- $\alpha$ (TNF- $\alpha$ ) were assessed using mouse ELISA kits (IL-2, F10780; IL-6, F10830; HGF, F10610; IFN- $\gamma$, F10660; IL-10, F10870; PEG2, F11430; TGF- $\beta 1$, F11590; TNF- $\alpha$, F11630; Shanghai Westang Bio-Tech, Co., Ltd., Shanghai, China) according to the manufacturer's instructions.

miRNA sequencing. The spleens were quickly removed following sacrifice of the mice. Splenic lymphocytes were purified by Ficoll density gradient centrifugation using Mouse 1X Lymphocyte Separation Medium (Dakewe Biotech, Co., Ltd., Beijing, China) and stained with fluorescein isothiocyanate anti-mouse CD4 (1:500 dilution; cat. no., 100509; BioLegend, Inc., San Diego, CA, USA) at $4^{\circ} \mathrm{C}$ for $30 \mathrm{~min}$. Using flow cytometry (BD FACSCalibur; BD Biosciences, Franklin Lakes, NJ, USA) CD4 ${ }^{+}$cells were sorted. Total RNA from $\mathrm{CD} 4^{+} \mathrm{T}$ cells of each sample was extracted using TRIzol reagent according to the manufacturer's protocol (Qiagen $\mathrm{GmbH}$, Hilden, Germany). The concentration of the RNA was measured using a NanoDrop 2000 spectrophotometer (Thermo Fisher Scientific, Inc., Wilmington, DE, USA) and RNA integrity was assessed using a Bioanalyzer 2100 (Agilent Technologies, Inc., Santa Clara, CA, USA). Total RNA (1 $\mu \mathrm{g})$ was used to prepare small RNA libraries for deep sequencing according to the manufacturer's instructions (HiSeq 3000; Illumina, Inc., San Diego, CA, USA). Raw sequencing data was mapped to a mouse miRNA database (miRBase version 20; http://www.mirbase.org) using miRanalyzer and bowtie 1.1.0 (http://bowtie-bio.sourceforge.net/index.shtml).
Reverse transcription-quantitative polymerase chain reaction (RT- $q P C R)$. After the RNA samples of splenic $\mathrm{CD} 4^{+} \mathrm{T}$ cells were used for deep sequencing, the remaining samples underwent RT-qPCR. cDNA was synthesized from $200 \mathrm{ng}$ total RNA using the TaqMan ${ }^{\circledR}$ MicroRNA reverse transcription kit (Applied Biosystems; Thermo Fisher Scientific, Inc.). cDNA was amplified using TaqMan microRNA Assay primers and the TaqMan Universal PCR Mastermix (Thermo Fisher Scientific, Inc.), according to the manufacturer's instructions. The primers for miR-155 were: Forward, 5'-AATGCTAAT CGTGATAGGGGT-3' and reverse, 5'-GTCTTCTTGGTG GCGAGGC-3'. The primers for U6 snRNA (used as a control) were: Forward, 5'-GATGACACGCAAATTGGTGAAG-3' and reverse, 5'-ATCTGGTTCGTGGAGTAGGAT-3'. The amplification reactions were carried out using a QuantStudio 3 Real-Time PCR system (Thermo Fisher Scientific, Inc.) with an initial hold step $\left(95^{\circ} \mathrm{C}\right.$ for $\left.5 \mathrm{~min}\right)$ and 50 cycles of a three-step PCR $\left(95^{\circ} \mathrm{C}\right.$ for $15 \mathrm{sec}, 60^{\circ} \mathrm{C}$ for $15 \mathrm{sec}$ and $72^{\circ} \mathrm{C}$ for $30 \mathrm{sec}$ ). Levels of relative miRNA expression were calculated by normalization to endogenous U6 snRNA expression. The fold changes were calculated using the $2^{-\Delta \Delta \mathrm{Cq}}$ method (31).

Statistical analysis. Data are presented as the mean \pm standard deviation. Differences were evaluated using one-way analysis of variance (least significant difference $t$-test) for multiple comparisons. Comparisons between different time points were performed using the paired $t$-test. All analyses were performed using SPSS 20.0 (IBM SPSS, Armonk, NY, USA) and statistical significance was set at $\mathrm{P}<0.05$.

\section{Results}

Water consumption and blood glucose of NOD mice increases as the age of the mice increases. Daily water consumption of NOD mice was measured when mice were aged between 10 and 25 weeks. Blood glucose of NOD mice was measured when mice were aged between 10 and 24 weeks. Daily water consumption of NOD mice remained stable prior to week 16, then gradually increased (Fig. 1A). The same effect was observed regarding blood glucose levels (Fig. 1B). Increased blood glucose levels and water consumption partly reflects the disease severity of SS in NOD mice. These results indicated 


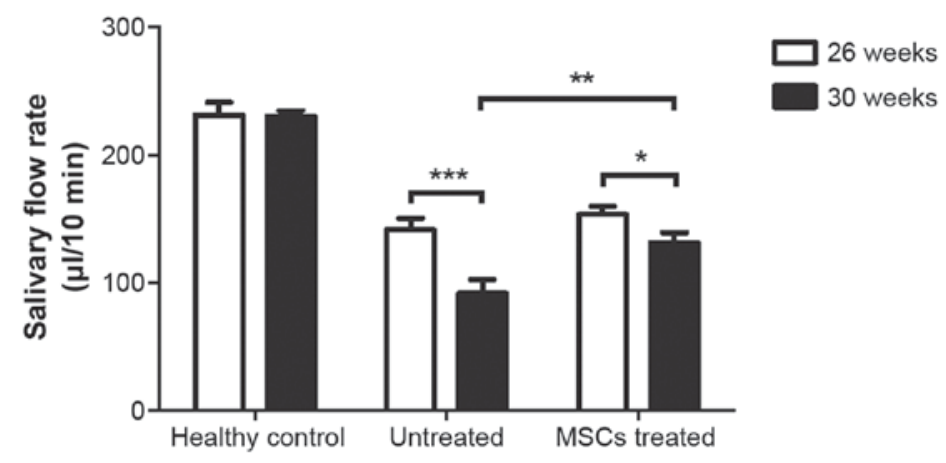

Figure 2. MSC treatment prevented the reduction of NOD mice salivary flow rate. Salivary flow rate of the three groups of mice were measured at the age of 26 weeks. Then, the treatment group received two MSC injections per week for 2 weeks. At 2 weeks after the MSC therapy finished (when mice were 30 weeks old), the salivary flow rates of the three groups of mice were measured again. The results show that, compared with the untreated group, the treatment group had a lower reduction of salivary flow rate (from 26 to 30 weeks of age). ${ }^{*} \mathrm{P}<0.05,{ }^{* * *} \mathrm{P}<0.01,{ }^{* * *} \mathrm{P}<0.001$. NOD mice, non-obese diabetic mice; MSC, mesenchymal stem cell.

A

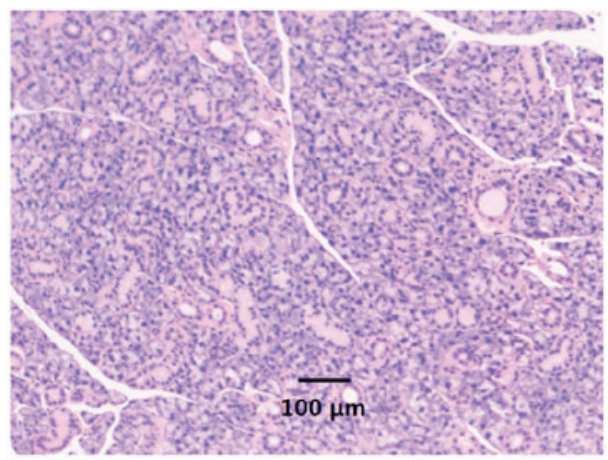

$\mathrm{C}$

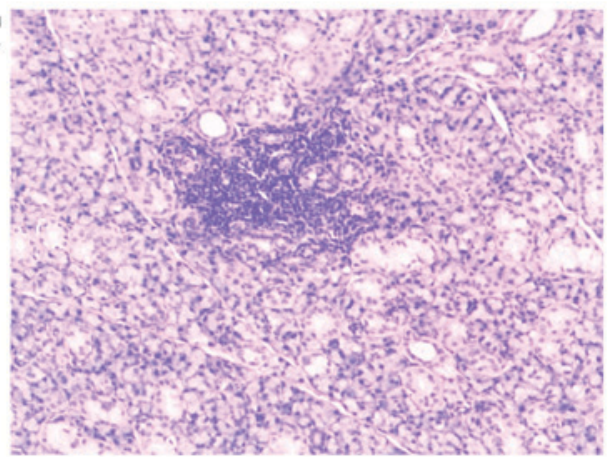

B

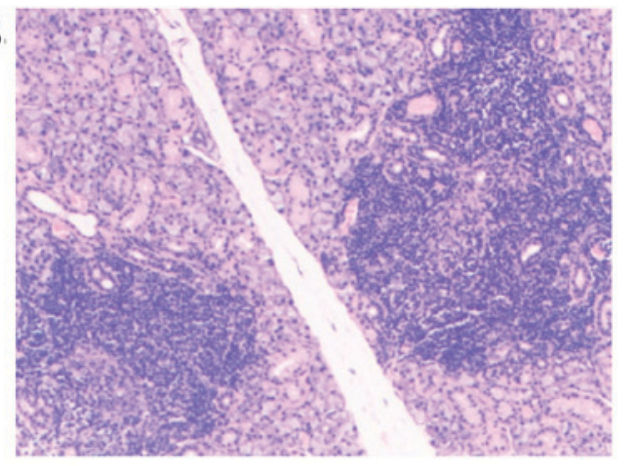

D

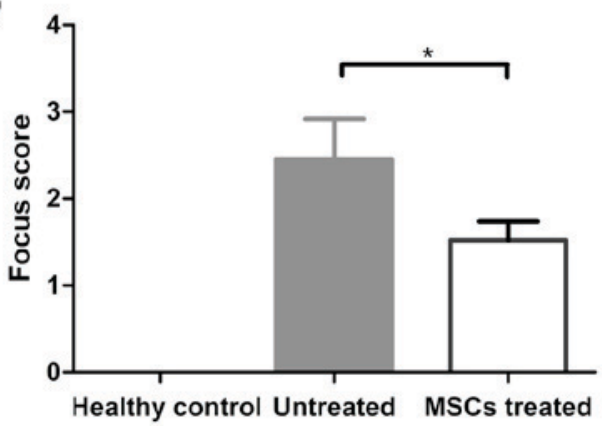

Figure 3. MSC-treated NOD mice have lower lymphocyte infiltration in salivary glands than untreated NOD mice. Hematoxylin and eosin staining of the submandibular glands of (A) healthy control, (B) untreated NOD and (C) MSC-treated NOD mice (magnification, $x 400$ ). (D) Focus score of the three groups mice. The focus score of healthy mice was 0 and the focus score was significantly lower in treatment group than untreated group $\left({ }^{*} \mathrm{P}=0.01\right.$; $\left.\mathrm{n}=8\right)$. NOD mice, non-obese diabetic mice. MSC, mesenchymal stem cells.

that the disease of NOD mice continued to deteriorate as the age of mice increased and that NOD mice had relatively severe SS when they were 25 weeks old.

$B M-M S C$ therapy reduces the decline in salivary secretion of NOD mice. SS-like autoimmune disorders usually appear when NOD/Ltj mice are aged 7-8 weeks old (22). To investigate whether BM-MSCs have therapeutic effects on relatively late stage SS-like inflammation in mice, MSCs were infused into NOD/Ltj mice when they reached 26 weeks old. Salivary gland function was assessed by measuring the salivary flow rates of NOD mice 1 day prior to and 2 weeks following MSC therapy. The salivary flow rates of age-matched untreated NOD mice and healthy control ICR mice were also measured. Salivary flow rates of healthy control ICR mice remained almost unchanged when mice were between 26 and 30 weeks of age $(\mathrm{P}=0.933)$. However, during this same period, salivary flow rates of untreated NOD mice declined rapidly $(\mathrm{P}=0.001)$. In the treatment group, mice that received MSC infusions exhibited less reduced saliva flow rates $(\mathrm{P}=0.039,30$ weeks vs. 26 weeks of age) than the age-matched untreated group (Fig. 2). These results indicated that BM-MSC treatment was effective in delaying the deterioration of the secretory function of salivary glands in mice with relatively late stage SS. 
A

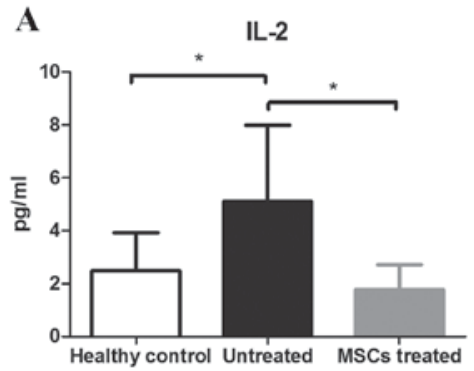

C

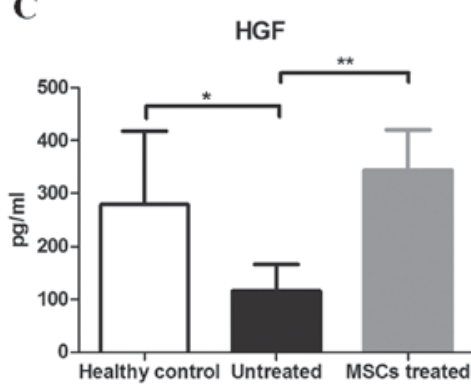

E

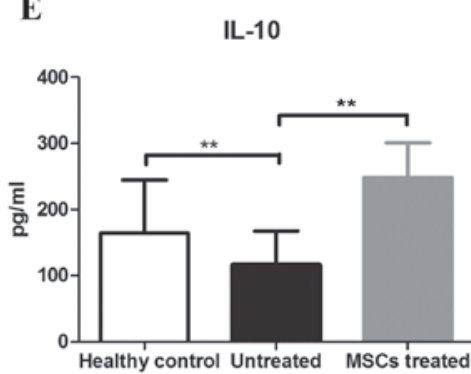

G

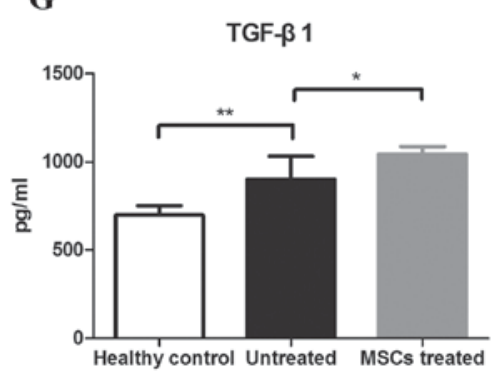

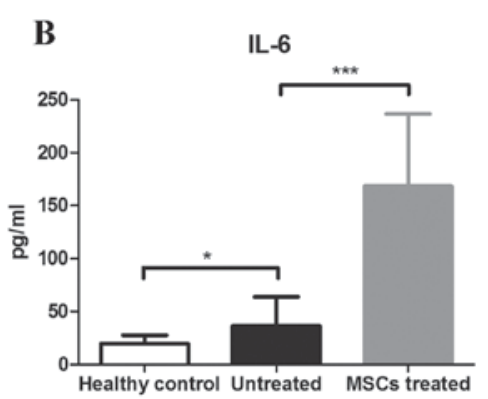

D

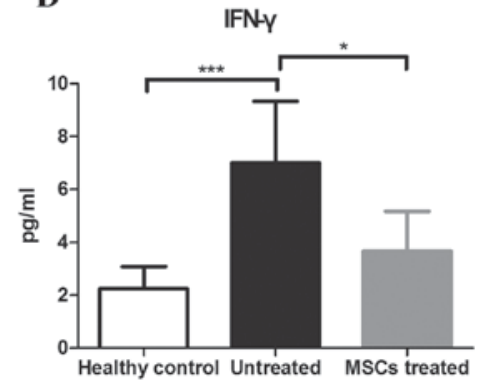

F

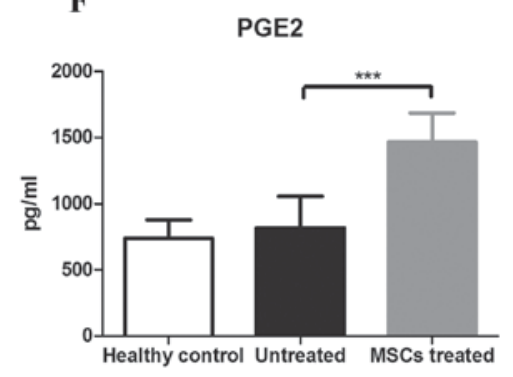

$\mathbf{H}$

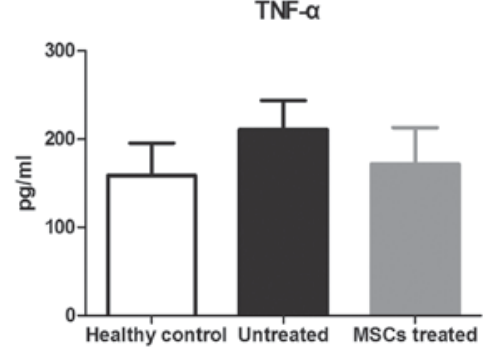

Figure 4. Concentration of key inflammatory cytokines and growth factors involved in the pathogenesis of Sjögren's syndrome or the immune modulating function of MSCs. The three groups of mice were sacrificed and the serums were obtained to measure the concentration of cytokines and growth factors at the age of 30 weeks (n=8). (A) IL-2, (B) IL-6, (C) HGF, (D) IFN- $\gamma$, (E) IL-10, (F) PEG2, (G) TGF- $\beta 1$ and (H) TNF- $\alpha .{ }^{*} \mathrm{P}<0.05,{ }^{* * *} \mathrm{P}<0.01,{ }^{* * * *} \mathrm{P}<0.001$. IL, interleukin; HGF, hepatocyte growth factor; IFN- $\gamma$, interferon $\gamma$; PEG2, prostaglandin E2; TGF- $\beta 1$, transforming growth factor $\beta 1$; TNF- $\alpha$, tumor necrosis factor $\alpha$; MSC, mesenchymal stem cells.

BM-MSC-treated NOD mice have lower lymphocyte infiltration in salivary glands than untreated NOD mice. Following MSC treatment for 2 weeks, the three groups of mice were sacrificed and salivary glands were histologically analyzed for lymphocyte infiltration. The results demonstrate that there was no significant lymphocyte infiltration in the salivary glands of healthy control mice, while lymphocyte infiltration was detected in the salivary glands of treated and untreated mice (Fig. 3A-C). The focus score in the salivary glands were $0 \pm 0$, $2.44 \pm 0.48$ and $1.52 \pm 0.66$ in the healthy control, treatment and untreated groups, respectively. The focus score of treated mice was significantly lower than that of untreated mice $(\mathrm{P}=0.01$; Fig. 3D). These results demonstrated that BM-MSC therapy alleviates lymphocyte infiltration in the salivary glands of NOD mice.

BM-MSC therapy alters concentrations of serum cytokines/growth factors in NOD mice. The expression levels of important cytokines/growth factors in the serum involved in the pathogenesis of SS and/or MSC immune modulating function were evaluated. Levels of IL-2 in the healthy, untreated and treatment groups were $2.478 \pm 1.444$, $5.114 \pm 2.870$ and $1.783 \pm 0.939 \mathrm{pg} / \mathrm{ml}$, respectively, $(\mathrm{F}=3.952$, $\mathrm{P}=0.046)$. IL-2 levels were significantly lower in the healthy and treated groups compared with the untreated group ( $\mathrm{P}=0.044, \mathrm{P}=0.025$, respectively; Fig. 4A). IL-6 levels in the 
Table I. Differentially expressed miRNAs in treated vs. untreated group.

\begin{tabular}{|c|c|c|c|}
\hline miRNA & $\begin{array}{l}\log _{2} \text { fold } \\
\text { change }\end{array}$ & P-value & $\begin{array}{l}\text { Change in expression in } \\
\text { treated vs. untreated group }\end{array}$ \\
\hline mmu-miR-669d-5p & 1.2190 & 0.0041 & Up \\
\hline mmu-miR-669b-5p & 1.0769 & 0.0120 & Up \\
\hline mmu-miR-615-3p & 1.0592 & 0.0153 & Up \\
\hline mmu-miR-20a-5p & 0.9230 & $<0.0001$ & Up \\
\hline mmu-miR-155-3p & 0.8484 & 0.0373 & Up \\
\hline mmu-miR-669o-5p & 0.8339 & 0.0370 & Up \\
\hline mmu-miR-19b-3p & 0.8022 & $<0.0001$ & Up \\
\hline mmu-miR-455-5p & 0.7784 & 0.0377 & Up \\
\hline mmu-miR-92a-1-5p & 0.7456 & 0.0088 & Up \\
\hline mmu-miR-26b-5p & 0.7097 & 0.0018 & Up \\
\hline mmu-miR-5122 & -1.5247 & $<0.0001$ & Down \\
\hline mmu-miR-6516-3p & -1.4882 & 0.0005 & Down \\
\hline mmu-miR-690 & -1.2844 & $<0.0001$ & Down \\
\hline mmu-miR-1940 & -1.2565 & 0.0023 & Down \\
\hline mmu-miR-5128 & -1.2346 & $<0.0001$ & Down \\
\hline mmu-miR-1843a-3p & -1.2085 & $<0.0001$ & Down \\
\hline mmu-miR-1895 & -1.1643 & 0.0008 & Down \\
\hline mmu-miR-677-3p & -1.1413 & 0.0087 & Down \\
\hline mmu-miR-6538 & -1.1409 & 0.0012 & Down \\
\hline mmu-miR-466a-3p & -1.0967 & 0.0032 & Down \\
\hline
\end{tabular}

Up, upregulated; Down, downregulated; miR/miRNA, microRNA.

healthy, untreated and treated groups were 19.770 \pm 7.969 , $36.619 \pm 27.114$ and $168.357 \pm 68.370 \mathrm{pg} / \mathrm{ml}$, respectively $(\mathrm{F}=21.570, \mathrm{P}<0.001)$. IL-6 levels were significantly higher in the treated group compared with the untreated and healthy groups (both $\mathrm{P}<0.01$; Fig. 4B). Levels of HGF in the healthy, untreated and treated groups were $279.319 \pm 138.650$, $115.487 \pm 50.539$ and $343.949 \pm 76.626 \mathrm{pg} / \mathrm{ml}$, respectively $(\mathrm{F}=7.448, \mathrm{P}=0.007)$. HGF levels were higher in the healthy and treated groups compared with the untreated group $(\mathrm{P}=0.013, \mathrm{P}=0.003$, respectively; Fig. 4C). Levels of IFN $-\gamma$ in the healthy, untreated and treated groups were 2.250 \pm 0.834 , $7.004 \pm 2.332$ and $3.678 \pm 1.499 \mathrm{pg} / \mathrm{ml}$, respectively, $(\mathrm{F}=12.254$, $\mathrm{P}=0.001)$. IFN- $\gamma$ levels were lower in healthy and treated groups compared with the untreated group $(\mathrm{P}<0.001, \mathrm{P}=0.01$, respectively; Fig. 4D). IL-10 levels in the healthy, untreated and treated groups were $164.163 \pm 80.826,117.226 \pm 50.365$ and $248.821 \pm 51.926 \mathrm{pg} / \mathrm{ml}$, respectively $(\mathrm{F}=5.070, \mathrm{P}=0.024)$. Compared with the untreated group, IL-10 levels were higher in the treated group ( $\mathrm{P}=0.007$; Fig. 4E). Levels of prostaglandin E2 (PGE2) of the healthy, untreated and treated groups were $739.272 \pm 137.522,819.115 \pm 235.885$ and $1,465.912 \pm 220.510 \mathrm{pg} / \mathrm{ml}$, respectively $(\mathrm{F}=17.970, \mathrm{P}<0.001)$. PGE2 levels were higher in the treated group than the untreated and healthy groups (both $\mathrm{P}<0.001$; Fig. 4F). Levels of TGF- $\beta 1$ of the healthy, untreated and treated groups were $697.718 \pm 54.542,904.892 \pm 66.593$ and $1,044.135 \pm 41.781 \mathrm{pg} / \mathrm{ml}$, respectively $(\mathrm{F}=19.770, \mathrm{P}<0.001)$. Compared with the healthy group, TGF- $\beta 1$ levels were higher in the untreated and treated groups $(\mathrm{P}=0.001, \mathrm{P}<0.001$, respectively). Furthermore, $\mathrm{TGF}-\beta 1$ levels were significantly higher in the treated group than the untreated group ( $\mathrm{P}=0.029$; Fig. 4G). Levels of TNF- $\alpha$ of the healthy, untreated and treated groups were $159.112 \pm 36.512$, $211.488 \pm 32.401$ and $171.705 \pm 41.515 \mathrm{pg} / \mathrm{ml}$, respectively, $(\mathrm{F}=3.342, \mathrm{P}=0.067)$. There were no significant differences in TNF- $\alpha$ levels among any of the groups (Fig. $4 \mathrm{H}$ ).

Differentially expressed miRNA profiles of mice splenic CD4 ${ }^{+}$ $T$ cells isolated from healthy, untreated and treated groups. Three samples of each group, randomly selected using a random number table, underwent deep sequencing. A total of 1,247 types of miRNAs were obtained and analyzed to determine whether they met the pre-determined criteria: Fold change $>1.5$ and $\mathrm{P}<0.05$. It was observed that 24 miRNAs were upregulated and $35 \mathrm{miRNAs}$ were downregulated in the treated group compared with the untreated group. The top 10 most upregulated and downregulated miRNAs in the treated group compared with the untreated group are presented respectively (Table I). Furthermore, the results demonstrated that 176 miRNAs were upregulated and 193 miRNAs were downregulated in the untreated group compared with the healthy group. The top 10 most upregulated and downregulated miRNAs in the untreated group compared with the healthy group are presented (Table II). To validate the deep sequencing results, RT-qPCR was performed to evaluate the expression of miR-155, which is known to be overexpressed in the PBMCs of patients with SS (27). The results were in agreement with the deep sequencing data (Table III), indicating the deep sequencing data were reliable. 
Table II. Differentially expressed miRNAs in healthy group vs. untreated group.

\begin{tabular}{lccc}
\hline & $\begin{array}{c}\text { Log }_{2} \text { fold } \\
\text { change }\end{array}$ & P-value & $\begin{array}{c}\text { Change in expression in } \\
\text { untreated vs. healthy groups }\end{array}$ \\
\hline mmu-miR-8115 & 6.2790 & $<0.0001$ & $\mathrm{Up}$ \\
mmu-miR-205-5p & 5.1677 & $<0.0001$ & $\mathrm{Up}$ \\
mmu-miR-6905-5p & 4.7022 & $<0.0001$ & $\mathrm{Up}$ \\
mmu-miR-486-5p & 4.5860 & $<0.0001$ & $\mathrm{Up}$ \\
mmu-miR-7016-3p & 4.1955 & 0.0019 & $\mathrm{Up}$ \\
mmu-miR-486-3p & 4.1100 & $<0.0001$ & $\mathrm{Up}$ \\
mmu-miR-672-5p & 4.0414 & 0.0030 & $\mathrm{Up}$ \\
mmu-miR-702-3p & 3.7167 & $<0.0001$ & $\mathrm{Up}$ \\
mmu-miR-7669-3p & 3.6666 & 0.0085 & $\mathrm{UP}$ \\
mmu-miR-1940 & 3.6458 & $<0.0001$ & $\mathrm{Up}$ \\
mmu-miR-3060-3p & $<.0001$ & Down \\
mmu-miR-455-5p & -6.1096 & $<0.0001$ & Down \\
mmu-miR-33-5p & -6.0665 & $<0.0001$ & Down \\
mmu-miR-542-3p & -6.0034 & $<0.0001$ & Down \\
mmu-miR-19a-5p & -5.9826 & $<0.0001$ & Down \\
mmu-miR-450b-5p & -5.3607 & $<0.0001$ & Down \\
mmu-miR-219a-5p & -4.9794 & $<0.0001$ & Down \\
mmu-miR-669d-5p & -4.8828 & $<0.0001$ & Down \\
mmu-miR-142-3p & -4.8656 & $<0.0001$ & Down \\
mmu-miR-136-5p & -4.6983 & 0.0003 & Down \\
\hline
\end{tabular}

Up, upregulated; down, downregulated; miR/miRNA, microRNA.

Table III. Relative expression of miR-155.

\begin{tabular}{lccc}
\hline Technique & $\begin{array}{c}\text { Untreated } \\
\text { group }\end{array}$ & $\begin{array}{c}\text { Healthy } \\
\text { group }\end{array}$ & $\begin{array}{c}\text { Treatment } \\
\text { group }\end{array}$ \\
\hline RT-qPCR & 1 & 0.471 & 1.210 \\
Deep sequencing & 1 & 0.809 & 1.292 \\
\hline
\end{tabular}

RT-qPCR, reverse transcription-quantitative polymerase chain reaction; miR, microRNA.

Functional analysis of differentially expressed miRNAs. To investigate the functional consequences of the differentially expressed miRNAs and their regulatory networks, Diana mirPath software (v.2.0; http://diana.imis.athena-innovation. $\mathrm{gr} /$ DianaTools/index.php?r=mirpath/index) was utilized to assess the molecular pathways potentially constituted by the miRNAs predicted target gene. Based on the microT-CDS database and using a threshold of $\mathrm{P}<0.05$ and MicroT $>0.8$, significantly enriched Kyoto Encyclopedia of Genes and Genomes (KEGG pathways) associated with differentially expressed miRNAs of the treated vs. untreated group were obtained (Fig. 5).

\section{Discussion}

A number of studies have demonstrated that MSCs have a therapeutic effect on NOD mice that received injections of MSCs between the ages of 7 and 16 weeks (22-24). SS-like autoimmune disorders usually appear in 7-8-week-old NOD mice (22); therefore, MSC therapy seems to be effective when mice are in the relatively early stage of SS. The dry eyes and mouth that occur in SS are attribute to the destruction of lacrimal and salivary glands following lymphocyte infiltration (32). MSCs exert a therapeutic effect by regulating lymphocytes to protect salivary glands $(22,23)$. In the late stage of SS, a large section of the gland has been destroyed, therefore it is unclear whether NOD mice with relatively late stage SS benefit from MSCs therapy. Determining whether this is the case may enable the development of therapeutic strategies to treat patients with late-stage SS. Therefore, the current study used 26-week-old NOD mice with SS as a mouse model of relatively late stage SS. Water intake and blood glucose levels of NOD mice were monitored when they were between 10 and 26 weeks old, and the continuously increased blood glucose levels and water consumption indicated continuous SS-like deterioration of the NOD mice.

The salivary flow rate can represent the salivary gland function. In the current study, the salivary flow rates of MSC-treated, age-matched healthy control and untreated mice were measured before and 2 weeks after MSC treatment. Treated and untreated NOD mice had lower salivary flow rates than healthy control mice at 26 and 30 weeks, indicating that they had impaired salivary gland function. The salivary flow rates of untreated NOD mice decreased significantly between 26 and 30 weeks $(\mathrm{P}<0.001)$, whereas the salivary flow rates of treated NOD mice showed a smaller decrease $(\mathrm{P}<0.05)$ over 
A
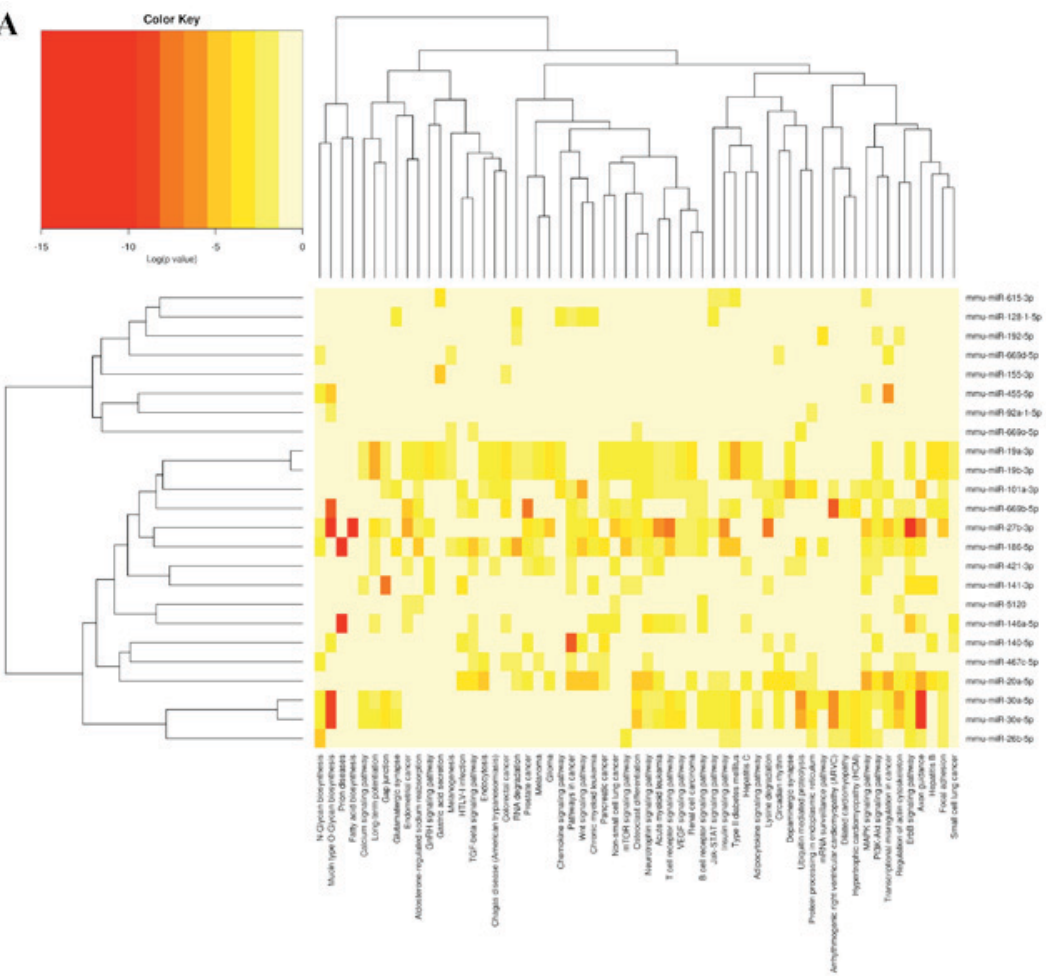

B
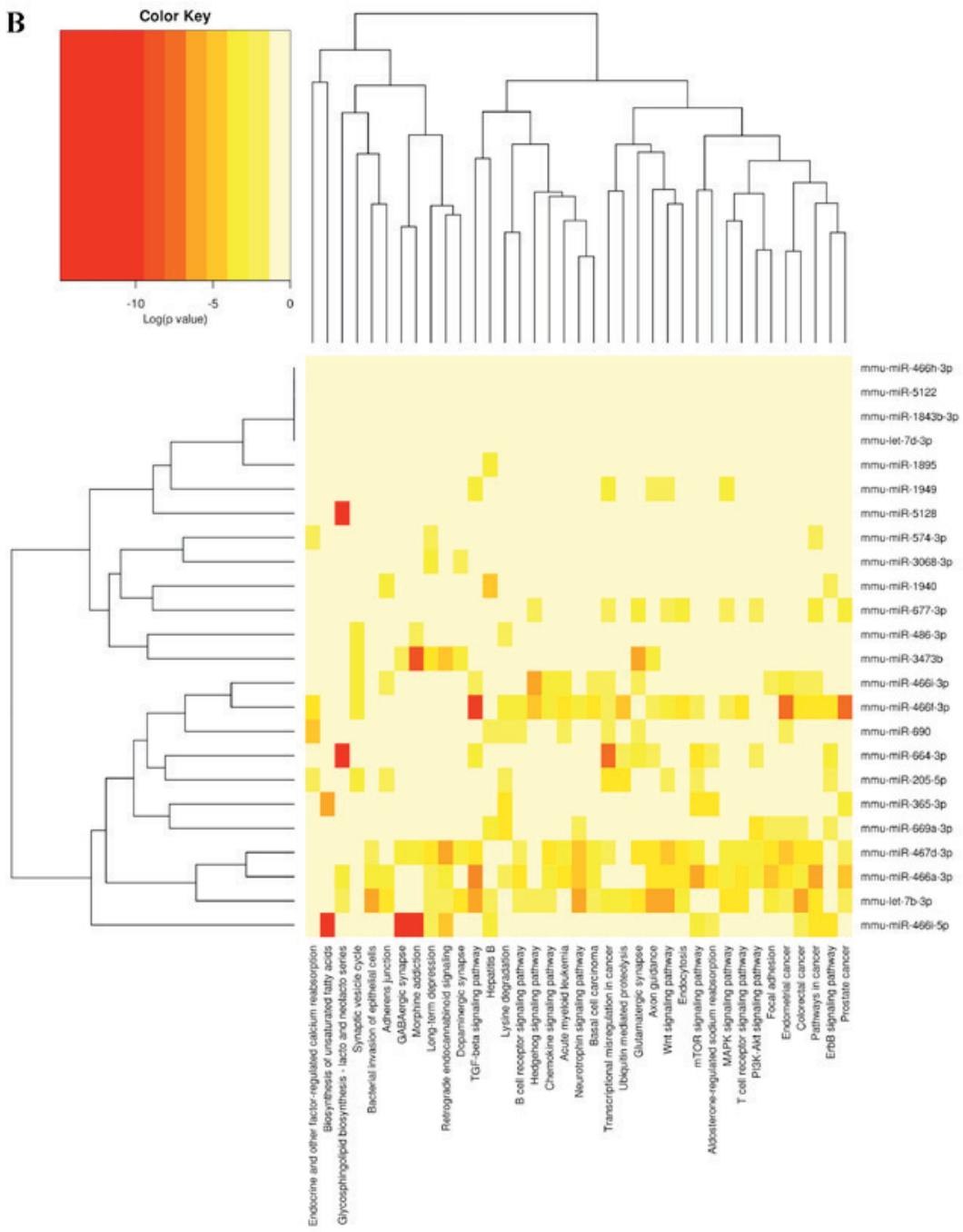

Figure 5. Heatmap of differentially expressed miRNAs of treatment group vs. untreated group and their associated KEGG pathways. (A) Upregulated miRNAs and their associated KEGG pathways. (B) Downregulated miRNAs and their associated KEGG pathways. miRNA, microRNA; KEGG, Kyoto Encyclopedia of Genes and Genomes. 
the same period. This result demonstrates that NOD mice with late stage SS may also benefit from MSC treatment. Previous studies, which treated the NOD mice with MSCs at an earlier stage than the current study, showed that MSC treatment can reverse the decline of NOD mice salivary flow rates (22-24). The results of the current study showed MSCs treatment only reduces the decline of salivary flow rates compared with untreated NOD mice, but does not reverse it. This difference in results may be explained by the fact that at the early stage of SS, lymphocyte infiltration only impairs the function of salivary gland cells but does not kill them; therefore, following the regulation of lymphocytes by MSC injection, the function of salivary gland cells is restored. However, in the relatively late stage of SS, a large number of salivary gland cells have already died, so MSC therapy can only protect residual salivary gland cells not already destroyed by lymphocytes. This difference suggests that patients with SS may experience more effective treatment with MSCs or other immunosuppressive therapies in the early stage of SS than at later stages. The current study measured the focus score in the salivary glands of the three groups of mice. The focus score of treated mice was lower than in the untreated mice but higher than in healthy control mice. This suggests that MSC therapy may have a slight curative effect on NOD mice with relatively late stage SS.

It has been reported that certain cytokines/growth factors are involved in the pathogenesis of SS and/or MSC immunomodulatory functions $(33,34)$. Therefore, in the current study, these cytokines/growth factors were measured, to try to determine the therapeutic effect of MSCs in NOD mice. IL-10, HGF, PGE2 and TGF- $\beta 1$ are cytokines/growth factors thought to serve a part in the immunomodulatory functions of MSCs (35). In the current study, the level of these cytokines/growth factors in serum was found to be higher in the treated group compared with the untreated group, suggesting that MSCs exert immunomodulatory functions in SS mice. Certain pro-inflammatory cytokines, including INF- $\gamma$, TNF- $\alpha$ and IL-6, are overexpressed in patients with SS (36-38). The results of the current study showed that levels of serum IL-6 and INF- $\gamma$ were higher in the untreated group compared with the healthy group. TNF- $\alpha$ levels were slightly higher in the untreated control group compared with the healthy controls; however, this difference was not significant. This demonstrates that the animal model of SS used in the current study was reliable. Levels of INF- $\gamma$ and TNF- $\alpha$ were lower in treatment group compared with the untreated group, although the difference in TNF- $\alpha$ levels was not significant. However, it remains unknown why levels of serum IL-6 were significantly higher in the treatment group than the untreated group. This result is similar to that obtained in a study by Jurewicz et al (39). In general, IL-6 is known as a pro-inflammatory cytokine, which can promote the population expansion and activation of $\mathrm{T}$ cells, the differentiation of $\mathrm{B}$ cells and regulation of the acute-phase response (40). However, IL-6 is also involved in the inhibition of monocyte differentiation into dendritic cells that is stimulated by MSCs (18). Therefore, the overall function of IL-6 in MSC immunomodulation requires further study. IL-2 is a pleiotropic cytokine that can promote and suppress immune responses depending on the dose and type of target cell. Low levels of IL-2 selectively target Treg expansion to limit inflammation while high levels of IL-2 promote effector T cell differentiation $(41,42)$. In the present study, levels of serum IL-2 were higher in the untreated group compared with the healthy and treatment groups. However, the effect of higher levels of IL-2 remains unclear. The interplays between MSCs and lymphocytes in vivo are complex and the exact roles of the aforementioned mediators in MSC-mediated immunosuppression are only partially understood and require further study.

Differential miRNA expression has been identified in autoimmune diseases, suggesting that miRNA regulation may be involved in autoimmune disease development or prevention $(43,44)$. A striking feature of miRNAs is their evolutionary conservation (45). Therefore, miRNA sequencing was used to identify the differentially expressed miRNAs of splenic CD4 ${ }^{+}$ $\mathrm{T}$ cells among groups. The results showed that the difference of splenic $\mathrm{CD}^{+} \mathrm{T}$ cell miRNA profiles between the treatment group and untreated group was not very evident. This may due to the migration of MSCs towards inflammatory salivary glands following injection to exert their immunoregulatory functions; therefore fewer MSCs are located in the spleen than in the salivary glands (22). The present study detected a significant difference in the concentration of cytokines/growth factors between the serum of treated and untreated groups, although this difference may be smaller than the difference in the level of cytokines/growth factors in the salivary glands of the two groups. Therefore, the alteration of splenic $\mathrm{CD}^{+} \mathrm{T}$ cell miRNA profiles between the two groups should exhibit the same trend but possibly to a lower extent compared with the alteration in the miRNA profiles of $\mathrm{CD}^{+} \mathrm{T}$ cells infiltrating the salivary glands. Among the top 10 most upregulated miRNAs identified in the treated group compared with the untreated group, miR-20a and miR-19b have been reported involved in immune-related diseases. Levels of miR-20a are significantly decreased in the blood of patients suffering from multiple sclerosis and this may inhibit $\mathrm{T}$ cell activation (46). Additionally, miR-19b is significantly decreased in the inflamed intestinal mucosa from patients with active Crohn's disease, and may reduce intestinal inflammation (47). In the current study, miR-20a-5p and miR-19b-3p were found to be underexpressed in the untreated group compared with the healthy group (6.250- and 11.166-fold lower than healthy group, respectively, both $\mathrm{P}<0.05$ ), indicating that miR-20a-5p and miR-19b-3p may also serve a role in the pathogenesis of $\mathrm{SS}$ and the immunoregulation functions of MSCs in $\mathrm{CD}^{+} \mathrm{T}$ cells.

The present study used ICR mice, which have a different genetic background to NOD mice, as healthy control mice. Therefore, the different miRNA profiles between the healthy and untreated groups may be explained by the different genetic background of the mice; however, some of the differentially expressed miRNAs between the two groups may serve roles in the pathogenesis of SS. It has been demonstrated that miR-155 serves an important role in regulating immune responses, and may affect the differentiation of $\mathrm{CD}^{+}{ }^{+} \mathrm{T}$ cells (48). Furthermore, it has been demonstrated that miR-155 is overexpressed in the PBMC of patients with SS (27). Therefore, to validate the reliability of the deep sequencing data, RT-qPCR was performed to evaluate miR-155 expression in the three groups. The results were in 
accordance with the deep sequencing data and showed that miR-155 was overexpressed in untreated groups compared with the healthy controls, which was consistent with the previous studies measuring miR-155 expression in patients with SS (27). While the results also showed that miR-155 was overexpressed in the treated group compared with the untreated group, indicating that MSCs may not exert therapeutic effects by regulating miR-155 of CD $4^{+} \mathrm{T}$ cells and the complexity of miRNA regulation.

In addition, bioinformatic analysis was used to predict the biological processes affected by differentially expressed miRNAs. The potential target genes of differentially expressed miRNAs between the treated and untreated groups were significantly enriched for a number of signaling pathways including the mitogen activated protein kinase, $\mathrm{T}$ cell receptor, Wnt, phosphatidylinositol 3-kinase-Akt, Jak-signal transducer and activator of transcription, mTOR and TGF- $\beta$ pathways. These pathways are known to regulate the activation, proliferation and differentiation of $\mathrm{CD}^{+} \mathrm{T}$ and are frequently dysregulated in autoimmune disease (49-61). The miRNA biological function prediction results implied that miRNAs may be involved in the MSC immunoregulation function of splenic $\mathrm{CD} 4^{+} \mathrm{T}$ cells; however, the exact molecular mechanism still requires identification.

In conclusion, MSCs have a therapeutic effect on relatively late stage SS in NOD mice. This effect may occur through alteration of the concentrations of serum cytokines/growth factors and the miRNA profile of $\mathrm{CD} 4^{+} \mathrm{T}$ cells in NOD mice. However, the underlying mechanisms require further study.

\section{References}

1. Mavragani CP and Moutsopoulos HM: The geoepidemiology of Sjögren's syndrome. Autoimmun Rev 9: A305-A310, 2010.

2. Fox RI: Sjögren's syndrome: Lancet 366: 321-331, 2005.

3. Ramos-Casals M, Tzioufas AG, Stone JH, Sisó A and Bosch X: Treatment of primary Sjögren syndrome: A systematic review. JAMA 304: 452-460, 2010.

4. Zhu J and Paul WE: CD4 T cells: Fates, functions, and faults. Blood 112: 1557-1569, 2008.

5. Busch R, Hadjinicolaou AV and Hall FC: Local activation and systemic dysregulation of T lymphocytes in Sjögren's syndrome. Curr Pharm Biotechnol 13: 2009-2021, 2012.

6. Altorok N, Coit P, Hughes T, Koelsch KA, Stone DU, Rasmussen A, Radfar L, Scofield RH, Sivils KL, Farris AD and Sawalha AH: Genome-wide DNA methylation patterns in naive $\mathrm{CD} 4^{+} \mathrm{T}$ cells from patients with primary Sjögren's syndrome. Arthritis Rheumatol 66: 731-739, 2014.

7. Sudzius G, Mieliauskaite D, Siaurys A, Viliene R, Butrimiene I, Characiejus D and Dumalakiene I: Distribution of peripheral lymphocyte populations in primary sjögren's syndrome patients. J Immunol Res 2015: 854706, 2015.

8. Sarigul M, Yazisiz V, Bassorgun CI, Ulker M, Avci AB, Erbasan F, Gelen T, Gorczynski RM and Terzioglu E: The numbers of Foxp $^{+}$Treg cells are positively correlated with higher grade of infiltration at the salivary glands in primary Sjogren's syndrome. Lupus 19: 138-145, 2010.

9. Yin $\mathrm{H}$, Zhao M, Wu X, Gao F, Luo Y, Ma L, Liu S, Zhang G, Chen J, Li F, et al: Hypomethylation and overexpression of CD70 (TNFSF7) in CD4 ${ }^{+} \mathrm{T}$ cells of patients with primary Sjögren's syndrome. J Dermatol Sci 59: 198-203, 2010.

10. Li XY, Wu ZB, Ding J, Zheng ZH, Li XY, Chen LN and Zhu P: Role of the frequency of blood CD4(+) CXCR5(+) CCR6(+) T cells in autoimmunity in patients with Sjögren's syndrome. Biochem Biophys Res Commun 422: 238-244, 2012.

11. Belkhir R, Gestermann N, Koutero M, Seror R, Tost J, Mariette X and Miceli-Richard C: Upregulation of membrane-bound CD40L on $\mathrm{CD}^{+} \mathrm{T}$ cells in women with primary Sjögren's syndrome. Scand J Immunol 79: 37-42, 2014.
12. Tran SD, Kodama S, Lodde BM, Szalayova I, Key S, Khalili S, Faustman DL and Mezey E: Reversal of Sjogren's-like syndrome in non-obese diabetic mice. Ann Rheum Dis 66: 812-814, 2007.

13. Bayetto K and Logan RM: Sjögren's syndrome: A review of aetiology, pathogenesis, diagnosis and management. Aust Dent J 55 (Suppl 1): S39-S47, 2010.

14. Jonsson MV, Delaleu N and Jonsson R: Animal models of Sjögren's syndrome. Clin Rev Allergy Immunol 32: 215-224, 2007.

15. Halleux C, Sottile V, Gasser JA and Seuwen K: Multi-lineage potential of human mesenchymal stem cells following clonal expansion. J Musculoskelet Neuronal Interact 2: 71-76, 2001.

16. Rosenbaum AJ, Grande DA and Dines JS: The use of mesenchymal stem cells in tissue engineering: A global assessment. Organogenesis 4: 23-27, 2008.

17. Nauta AJ and Fibbe WE: Immunomodulatory properties of mesenchymal stromal cells. Blood 110: 3499-3506, 2007.

18. Jiang XX, Zhang Y, Liu B, Zhang SX, Wu Y, Yu XD and Mao N: Human mesenchymal stem cells inhibit differentiation and function of monocyte-derived dendritic cells. Blood 105: 4120-4126, 2005.

19. Sotiropoulou PA, Perez SA, Gritzapis AD, Baxevanis CN and Papamichail M: Interactions between human mesenchymal stem cells and natural killer cells. Stem Cells 24: 74-85, 2006.

20. Ren G, Zhang L, Zhao X, Xu G, Zhang Y, Roberts AI, Zhao RC and Shi Y: Mesenchymal stem cell-mediated immunosuppression occurs via concerted action of chemokines and nitric oxide. Cell Stem Cell 2: 141-150, 2008.

21. Tse WT, Pendleton JD, Beyer WM, Egalka MC and Guinan EC: Suppression of allogeneic T-cell proliferation by human marrow stromal cells: Implications in transplantation. Transplantation 75: 389-397, 2003.

22. Xu J, Wang D, Liu D, Fan Z, Zhang H, Liu O, Ding G, Gao R, Zhang C, Ding Y, et al: Allogeneic mesenchymal stem cell treatment alleviates experimental and clinical Sjögren syndrome. Blood 120: 3142-3151, 2012.

23. Khalili S, Liu Y, Sumita Y, Blank D, Key S, Mezey E and Tran SD: Bone marrow cells are a source of undifferentiated cells to prevent Sjögren's syndrome and to preserve salivary glands function in the non-obese diabetic mice. Int J Biochem Cell Biol 42: 1893-1899, 2010.

24. Khalili S, Liu Y, Kornete M, Roescher N, Kodama S, Peterson A, Piccirillo CA and Tran SD: Mesenchymal stromal cells improve salivary function and reduce lymphocytic infiltrates in mice with Sjögren's-like disease. PLoS One 7: e38615, 2012.

25. Liu R, Su D, Zhou M, Feng X, Li X and Sun L: Umbilical cord mesenchymal stem cells inhibit the differentiation of circulating $\mathrm{T}$ follicular helper cells in patients with primary Sjögren's syndrome through the secretion of indoleamine 2,3-dioxygenase. Rheumatology (Oxford) 54: 332-342, 2015.

26. Bartel DP: MicroRNAs: Genomics, biogenesis, mechanism, and function. Cell 116: 281-297, 2004.

27. Pauley KM, Stewart CM, Gauna AE, Dupre LC, Kuklani R, Chan AL, Pauley BA, Reeves WH, Chan EK and Cha S: Altered miR-146a expression in Sjögren's syndrome and its functional role in innate immunity. Eur J Immunol 41: 2029-2039, 2011.

28. Shi H, Zheng LY, Zhang P and Yu CQ: miR-146a and miR-155 expression in PBMCs from patients with Sjögren's syndrome. J Oral Pathol Med 43: 792-797, 2014.

29. Gourzi VC, Kapsogeorgou EK, Kyriakidis NC and Tzioufas AG: Study of microRNAs (miRNAs) that are predicted to target the autoantigens Ro/SSA and La/SSB in primary Sjögren's Syndrome. Clin Exp Immunol 182: 14-22, 2015.

30. Konsta OD, Thabet Y, Le Dantec C, Brooks WH, Tzioufas AG, Pers JO and Renaudineau Y: The contribution of epigenetics in Sjögren's Syndrome. Front Genet 5: 71, 2014.

31. Livak KJ and Schmittgen TD: Analysis of relative gene expression data using real-time quantitative PCR and the 2(-Delta Delta C(T)) Method. Methods 25: 402-408, 2001.

32. Jonsson R, Vogelsang P, Volchenkov R, Espinosa A, WahrenHerlenius M and Appel S: The complexity of Sjögren's syndrome: Novel aspects on pathogenesis. Immunol Lett 141: 1-9, 2011.

33. Saraux A, Pers JO and Devauchelle-Pensec V: Treatment of primary Sjögren syndrome. Nat Rev Rheumatol 12: 456-471, 2016.

34. Castro-Manrreza ME and Montesinos JJ: Immunoregulation by mesenchymal stem cells: Biological aspects and clinical applications. J Immunol Res 2015: 394917, 2015.

35. Ben-Ami E, Berrih-Aknin S and Miller A: Mesenchymal stem cells as an immunomodulatory therapeutic strategy for autoimmune diseases. Autoimmun Rev 10: 410-415, 2011. 
36. Roescher N, Tak PP and Illei GG: Cytokines in Sjogren's syndrome: Potential therapeutic targets. Ann Rheum Dis 69: 945-948, 2010

37. Sada PR, Isenberg D and Ciurtin C: Biologic treatment in Sjögren's syndrome. Rheumatology (Oxford) 54: 219-230, 2015.

38. Coursey TG and de Paiva CS: Managing Sjögren's Syndrome and non-Sjögren Syndrome dry eye with anti-inflammatory therapy. Clin Ophthalmol 8: 1447-1458, 2014.

39. Jurewicz M, Yang S, Augello A, Godwin JG, Moore RF, Azzi J, Fiorina P, Atkinson M, Sayegh MH and Abdi R: Congenic mesenchymal stem cell therapy reverses hyperglycemia in experimental type 1 diabetes. Diabetes 59: 3139-3147, 2010.

40. Hunter CA and Jones SA: IL-6 as a keystone cytokine in health and disease. Nat Immunol 16: 448-457, 2015.

41. Ballesteros-Tato A: Beyond regulatory T cells: The potential role for IL-2 to deplete T-follicular helper cells and treat autoimmune diseases. Immunotherapy 6: 1207-1220, 2014.

42. Bayer AL, Pugliese A and Malek TR: The IL-2/IL-2R system: From basic science to therapeutic applications to enhance immune regulation. Immunol Res 57: 197-209, 2013.

43. Sonkoly E and Pivarcsi A: Advances in microRNAs: Implications for immunity and inflammatory diseases. J Cell Mol Med 13: 24-38, 2009.

44. Pauley KM, Cha S and Chan EK: MicroRNA in autoimmunity and autoimmune diseases. J Autoimmun 32: 189-194, 2009.

45. Stein LD, Bao Z, Blasiar D, Blumenthal T, Brent MR, Chen N, Chinwalla A, Clarke L, Clee C, Coghlan A, et al: The genome sequence of Caenorhabditis briggsae: A platform for comparative genomics. PLoS Biol 1: E45, 2003.

46. Cox MB, Cairns MJ, Gandhi KS, Carroll AP, Moscovis S, Stewart GJ, Broadley S, Scott RJ, Booth DR, Lechner-Scott J, et al: MicroRNAs miR-17 and miR-20a inhibit T cell activation genes and are under-expressed in MS whole blood. PLoS One 5: e12132, 2010

47. Cheng X, Zhang X, Su J, Zhang Y, Zhou W, Zhou J, Wang C, Liang H, Chen X, Shi R, et al: miR-19b downregulates intestinal SOCS3 to reduce intestinal inflammation in Crohn's disease. Sci Rep 5: 10397, 2015.

48. Seddiki N, Brezar V, Ruffin N, Lévy Y and Swaminathan S: Role of miR-155 in the regulation of lymphocyte immune function and disease. Immunology 142: 32-38, 2014.
49. Notarangelo LD: Immunodeficiency and immune dysregulation associated with proximal defects of $\mathrm{T}$ cell receptor signaling. Curr Opin Immunol 31: 97-101, 2014.

50. Ma J, Wang R, Fang X and Sun Z: $\beta$-catenin/TCF-1 pathway in $\mathrm{T}$ cell development and differentiation. J Neuroimmune Pharmacol 7: 750-762, 2012.

51. Xue HH and Zhao DM: Regulation of mature $\mathrm{T}$ cell responses by the Wnt signaling pathway. Ann N Y Acad Sci 1247: 16-33, 2012

52. Soejima K, Nakamura H, Tamai M, Kawakami A and Eguchi K: Activation of MKK4 (SEK1), JNK and c-Jun in labial salivary infiltrating $\mathrm{T}$ cells in patients with Sjögren's syndrome. Rheumatol Int 27: 329-333, 2007.

53. Patterson H, Nibbs R, McInnes I and Siebert S: Protein kinase inhibitors in the treatment of inflammatory and autoimmune diseases. Clin Exp Immunol 176: 1-10, 2014.

54. Dodeller F and Schulze-Koops H: The p38 mitogen-activated protein kinase signaling cascade in CD4 T cells. Arthritis Res Ther 8: 205, 2006.

55. Hawkins PT and Stephens LR: PI3K signalling in inflammation. Biochim Biophys Acta 1851: 882-897, 2015

56. Ball J, Archer S and Ward S: PI3K inhibitors as potential therapeutics for autoimmune disease. Drug Discov Today 19: $1195-1199,2014$

57. Christie D and Zhu J: Transcriptional regulatory networks for CD4 T cell differentiation. Curr Top Microbiol Immunol 381: $125-172,2014$

58. Gadina M: Janus kinases: An ideal target for the treatment of autoimmune diseases. J Investig Dermatol Symp Proc 16: S70-S72, 2013.

59. Chapman NM and Chi H: mTOR signaling, Tregs and immune modulation. Immunotherapy 6: 1295-1311, 2014.

60. Tu E, Chia PZ and Chen W: TGF $\beta$ in T cell biology and tumor immunity: Angel or devil? Cytokine Growth Factor Rev 25: 423-435, 2014

61. Shachar I and Karin N: The dual roles of inflammatory cytokines and chemokines in the regulation of autoimmune diseases and their clinical implications. J Leukoc Biol 93: 51-61, 2013. 